\title{
Permittivity of Porous Titanate Dielectrics
}

\author{
Zachary N. Wing, ${ }^{* \dagger}$ Brian Wang, and John W. Halloran** \\ Department of Materials Science and Engineering, University of Michigan, Ann Arbor, Michigan 48109
}

\begin{abstract}
Relative permittivities ranging from 12 to 100 were obtained from porous titania. Relative permittivities from 8 to 30 were obtained from porous calcium magnesium titanates. Porosity was introduced as microporosity by partial sintering, as $\mathbf{1 5} \boldsymbol{\mu m}$ diameter spherical pores with porogens, and by fabrication of a microcellular structure with cylindrical pores. The relative permittivity as a function of porosity was compared with effective media models. Permittivity could be fitted to the Bruggeman 1/3 power law over a porosity range from $5 \%$ to $78 \%$.
\end{abstract}

\section{Introduction}

$\mathrm{U}$ SUALLY, the dielectric permittivity is adjusted by changing the chemical composition of the ceramic. Here, we consider adjusting the permittivity by the controlled addition of porosity. We consider two materials: titania and a calcium magnesium titanate (CMT) dielectric. $\mathrm{TiO}_{2}$ is a desirable material for microwave applications because of its high permittivity $\left(\varepsilon_{\mathrm{r}}=100\right)$ and low loss $\left(\tan \delta \sim 10^{-5}\right)$; however, its large temperature coefficient of resonance $\tau_{\mathrm{f}}\left(450 \mathrm{ppm} /{ }^{\circ} \mathrm{C}\right)$ can limit its application. ${ }^{1} \mathrm{CMT}$ are also of interest for microwave applications. They consist of the high $\varepsilon_{\mathrm{r}} \mathrm{Ca}$ titanates and low $\varepsilon_{\mathrm{r}} \mathrm{Mg}$ titanates. Microwave dielectrics based on $\mathrm{MgTiO}_{3}$ typically have $\mathrm{CaTiO}_{3}$ mixed in to balance out their negative temperature coefficient $\left(\tau_{\mathrm{f}}\right){ }^{2}$ This occurs by a partial substitution of $\mathrm{Mg}$ by $\mathrm{Ca}^{3}$ Compositions within the $\mathrm{CaTiO}_{3}-\mathrm{MgTiO}_{3}-\mathrm{Mg}_{2} \mathrm{TiO}_{4}\left(\mathrm{CT}-\mathrm{MT}-\mathrm{M}_{2} \mathrm{~T}\right)$ phase system are of technological interest because of the wide range of dielectric constants available and low loss $\left(\tan \delta<1.5 \times 10^{-3}\right)$.

There are several methods used to create porous ceramics. The most common method is to use residual porosity from incomplete sintering. ${ }^{4}$ However, this method only yields up to $40 \%$ porosity, with pore size similar to the grain size. Another approach is to use a sacrificial pore former. This method involves selecting porogens, materials that are pyrolyzed to leave behind stable pores with the same size as the porogen. Common porogens are starch particles, graphite, and various polymer spheres or carbon particles. ${ }^{5,6} \mathrm{~A}$ porous dielectric is a composite between the host and air, and it is well known that adding porosity can yield a dielectric permittivity that is lower than the permittivity of the solid material. This is one strategy to achieve very low $\varepsilon_{\mathrm{r}}$ dielectrics for microelectronics. ${ }^{7-9}$ Controlled porosity within a monolithic piece allows for spatial variations in permittivity, or dielectric texturization, which has application in antenna design. ${ }^{10,11}$ Microwave band-gap filters and resonators can also take advantage of texturization to localize electromagnetic radiation. ${ }^{12}$ Also, with oriented (nonspherical) pores, it is possible to exploit or deny specific field polarizations through the creation of anisotropic permittivity. ${ }^{13}$ Thus, the purpose of this work is to demonstrate the techniques and effectiveness of

A. Bandyopadhyay — contributing editor

Manuscript No. 21327. Received January 5, 2006; approved August 2, 2006 This work was supported by the Defense Advanced Research Project under Grant No. N00173-01-1-G910.

*Member, American Ceramic Society.

**Fellow, American Ceramic Society.

${ }^{\dagger}$ Author to whom correspondence should be addressed. e-mail: wingzach@umich.edu controlling the relative permittivity. Note, however, that the spatial scale of the medium must be much larger than the porosity to apply effective medium models. The wavelength of the electromagnetic radiation must also be much larger than the size of the pores in the composites.

A mixture of two or more dielectric materials (or nondielectrics) is essentially a material composite. Numerous mixture laws and approximations have previously been developed to account for such parameters as particle size, geometry, orientation, etc. The simplest model consists of layers of dielectric that are oriented either parallel (Eq. (1)) or perpendicular (Eq. (2)) to the applied electric field ${ }^{14}$ :

$$
\begin{aligned}
& \varepsilon_{\mathrm{eff}}=v_{1} \varepsilon_{1}+v_{2} \varepsilon_{2} \\
& \frac{1}{\varepsilon_{\mathrm{eff}}}=\frac{v_{1}}{\varepsilon_{1}}+\frac{v_{2}}{\varepsilon_{2}}
\end{aligned}
$$

Here $\varepsilon_{\text {eff }}, \varepsilon_{1}$, and $\varepsilon_{2}$ denote the relative permittivity of the composite, inclusions, and matrix, respectively. The volume fraction is represented as $v_{1}$ and $v_{2}$ for the inclusion and matrix, respectively. These basic models form an upper and lower bound (Wiener bounds) on the effective permittivity of binary mixtures. ${ }^{15}$

A porous dielectric can be considered a composite consisting of two phases - one solid and one void. If the voids are much smaller than an incident wavelength, scattering will not occur and the material behaves as an "effective medium." The pores inside the matrix material can be modeled as inclusions within the host. The result is a two-phase composite consisting of a fully dense (phase 1) phase and a distribution of pores (phase 2). The parallel and perpendicular models are too simplistic for a porous material.

Numerous models have been developed to describe dielectric mixtures from numerical fits, analytic derivations, and stochastic methods. These are typically based on host-inclusion models assuming spherical pores. Three often-used models include Maxwell-Garnet (MG), Landauer-Bruggeman effective medium approximation (EMA), and Bruggeman 1/3 power law, Eqs. (3)-(5), respectively

Maxwell-Garnet:

$$
\varepsilon_{\mathrm{eff}}=\left[\frac{2 \varepsilon_{2}+\varepsilon_{1}+2 v\left(\varepsilon_{1}-\varepsilon_{2}\right)}{2 \varepsilon_{2}+\varepsilon_{1}-v\left(\varepsilon_{1}-\varepsilon_{2}\right)}\right]\left(\varepsilon_{2}\right)
$$

$E M A$ :

$$
v \frac{\varepsilon_{1}-\varepsilon_{\mathrm{eff}}}{\varepsilon_{1}+2 \varepsilon_{\mathrm{eff}}}+(1-v) \frac{\varepsilon_{2}-\varepsilon_{\mathrm{eff}}}{\varepsilon_{2}+2 \varepsilon_{\mathrm{eff}}}=0
$$

Bruggeman 1/3:

$$
\left(\frac{\varepsilon_{2}}{\varepsilon_{\mathrm{eff}}}\right)^{1 / 3}\left(\frac{\varepsilon_{1}-\varepsilon_{\mathrm{eff}}}{\varepsilon_{1}-\varepsilon_{2}}\right)=(1-v)
$$

Inherent in these mixture rules are certain assumptions. MG theory assumes the included spheres are isolated from each other 
and the diameter of inclusion is much less than the inclusion spacing. ${ }^{16}$ Landauer-Bruggeman EMA assumes no short-range order. ${ }^{1,18}$ Bruggeman $1 / 3$ power law assumes that for a given volume, there is a unique $\varepsilon_{\text {eff }}$, where an incremental change in volume percent inclusion, $\delta_{\mathrm{vol}}$, changes the effective permittivity by $\varepsilon_{\text {eff }}+\delta \varepsilon_{\text {eff. }}{ }^{16}$

In this paper, we consider porosities ranging from quite low values $(5 \mathrm{vol} \%)$ to quite high porosity $(78 \mathrm{vol} \%)$. Porosity is induced in three ways: (1) residual sintering porosity for micropores; (2) spherical porogens from addition of $15 \mu \mathrm{m}$ carbon microspheres; and (3) cylindrical pores $(>50 \mu \mathrm{m})$ in a microcellular ceramic.

\section{Experimental Procedure}

Titania samples were fabricated from a commercial $99.8 \%$ pure titanium dioxide powder (203-4, Ferro Electronic Materials, Penn Yan, NY) with a particle size of $1.1-1.3 \mu \mathrm{m}$. The CTMT- $\mathrm{M}_{2} \mathrm{~T}$ powder is a commercial powder (MCT-30, TransTech Inc., Adamstown, MD). The form of carbon used as a fugitive was $15 \mu \mathrm{m}$ glassy carbon microspheres (Sigradur K, HTW GmbH, Thierhaupten, Germany).

To prepare microporous samples, powder compacts were partially sintered. A set of pure $\mathrm{TiO}_{2}$ samples was pressed inside a lubricated steel die at $150 \mathrm{MPa}$. Samples were pressed into a cylindrical geometry to a green diameter of $12.8 \mathrm{~mm}$ and a green height of $3.4 \mathrm{~mm}$. Pellets were placed in a bed of sintered powder. Firing was performed in air and at various temperatures $\left(1150^{\circ}-1400^{\circ} \mathrm{C}\right)$ at a heating and cooling rate of $10^{\circ} \mathrm{C} / \mathrm{min}$.

A fugitive porogen was used to induce spherical pores using the glassy carbon microspheres shown in Fig. 1. The pores induced by the glassy carbon particles were found to be approximately the same size as the particles, which were $15 \pm 5 \mu \mathrm{m}$. The volume fraction of spherical pores was calculated from the volume added, using a mass and a density of $1.5 \mathrm{~g} / \mathrm{cm}^{3}$. The carbon and raw powders were wet mixed for $15 \mathrm{~min}$ in iso-propanol, and dried at $80^{\circ} \mathrm{C}$ in air. Powder pellets were pressed using the same procedure as the pure $\mathrm{TiO}_{2}$ samples. Carbon removal was based on thermo gravimetric analysis for pure glassy carbon microspheres and took place in air between $450^{\circ}$ and $640^{\circ} \mathrm{C}$. After carbon removal, all samples were sintered in air at a heating and cooling rate of $10^{\circ} \mathrm{C} / \mathrm{min}$. Glassy carbon $\mathrm{TiO}_{2}$ samples were sintered at $1200^{\circ} \mathrm{C}(1 \mathrm{~h})$. After sintering in air, the titania samples were white in color and retained the rutile structure. There was no evidence of partial reduction by the carbon. CMT samples with the carbon porogen were prepared similarly. However, they were fired separately in air and sintered at $1360^{\circ} \mathrm{C}(4$ h).

Microcellular solids with cylindrical pores were produced by compaction of thermoplastic filaments made from titania powder with a fugitive carbon core. These filaments were made via

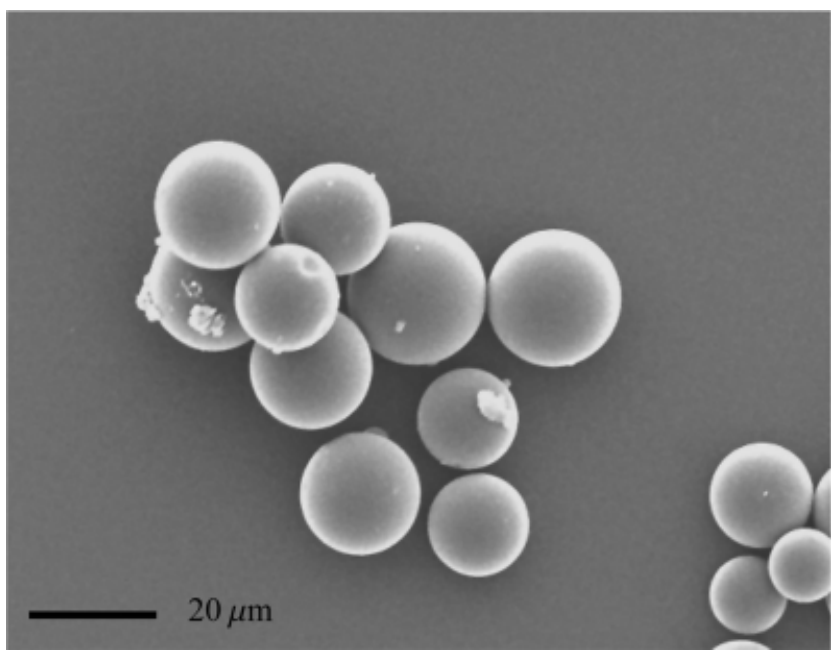

Fig. 1. Glassy carbon microspheres used as fugitive porogen.

the microfabrication by the co-extrusion (MFCX) process, which has been described in detail previously. ${ }^{19}$ Samples prepared using MFCX were compounded with an acrylate binder. A master feed rod was created by combining a $\mathrm{TiO}_{2}+$ binder shell (white) with a carbon black + binder core (black). The shell and core were warm bonded and co-extruded to a $3.25 \mathrm{~mm}$ rod having a titania shell and a carbon core (Bradford Small Scale Extrusion Unit, Bradford University Research Ltd., Bradford, U.K.). The $3.25 \mathrm{~mm}$ rods were bundled together as shown in Fig. 2, and co-extruded to a $270 \mu \mathrm{m}$ filament. Two types of samples were prepared using this technique. The first sample used a purely sacrificial core that left a hollow tube, and the second involved a partially sacrificial core containing 5-20 vol\% $\mathrm{TiO}_{2}$. Finally, the resulting fiber was chopped into $1-3 \mathrm{~mm}$ lengths to form a randomly-oriented structure. Chopped fibers were warm pressed at $125^{\circ} \mathrm{C}$ at $12 \mathrm{MPa}$ into cylinders. Binder removal was performed in air using a 3-day heating schedule based on previous TGA results. $\mathrm{MFCX} \mathrm{TiO}_{2}$ samples were sintered at $1300^{\circ} \mathrm{C}(2 \mathrm{~h})$. Porosity was measured using the Archimedes method.

The relative permittivities of powder-pressed samples were measured using an impedance analyzer and test fixture (HP 4194A and a $\pm 40 \mathrm{~V} 16047 \mathrm{D}$ fixture, Agilent Technologies Inc., Palo Alto, CA). Dielectric constants of sintered pellets were measured using a parallel-plate configuration. Pellets were ground to ensure flatness with 600 grit $\mathrm{SiC}$ paper. Typical samples were $10 \mathrm{~mm}$ in diameter and $2.8 \mathrm{~mm}$ in height. Top and bottom surfaces were metallized with silver paint (Ted Pella Leitsilber 200, Ted Pella Inc., Redding, CA) to serve as electrodes, taking care to assure that the silver paint did not get into
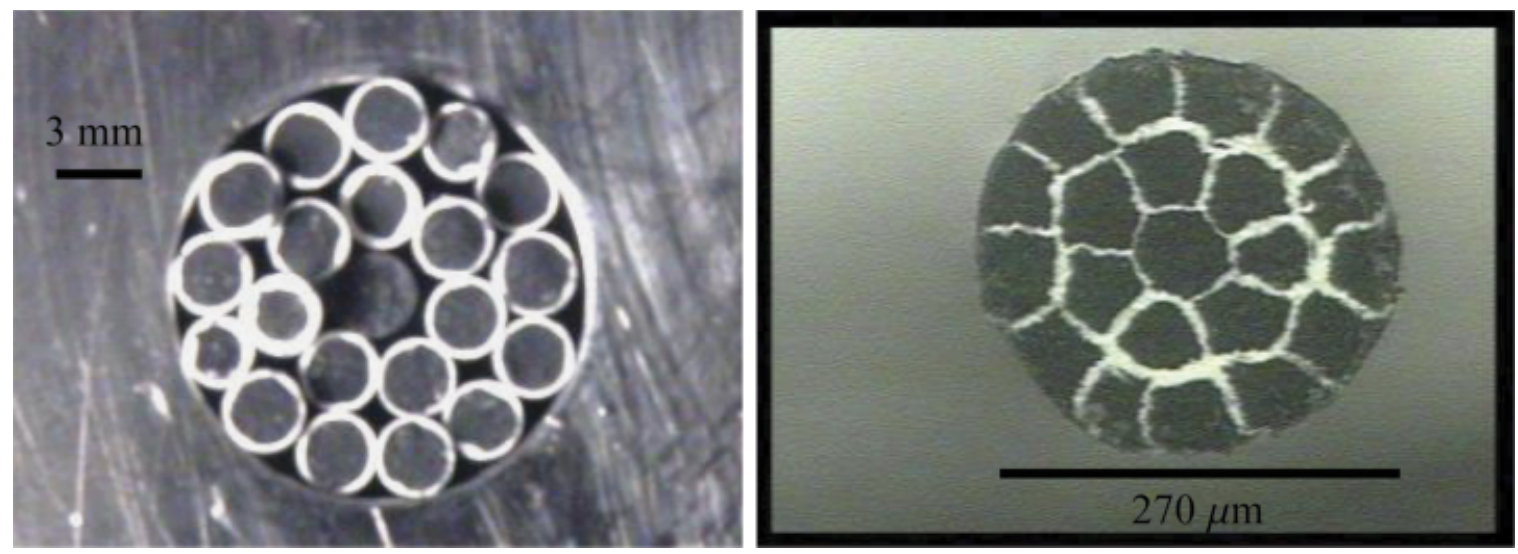

Fig. 2. (Left) Bundle of twenty $3.25 \mathrm{~mm}$ green filaments with a titania shell over a carbon core, around a single carbon filament. (Right) Resulting $270 \mu \mathrm{m}$ green filament after co-extrusion, forming a microcellular structure of titania (white) and fugitive carbon (black) (C) 2005 IEEE). ${ }^{13}$ 


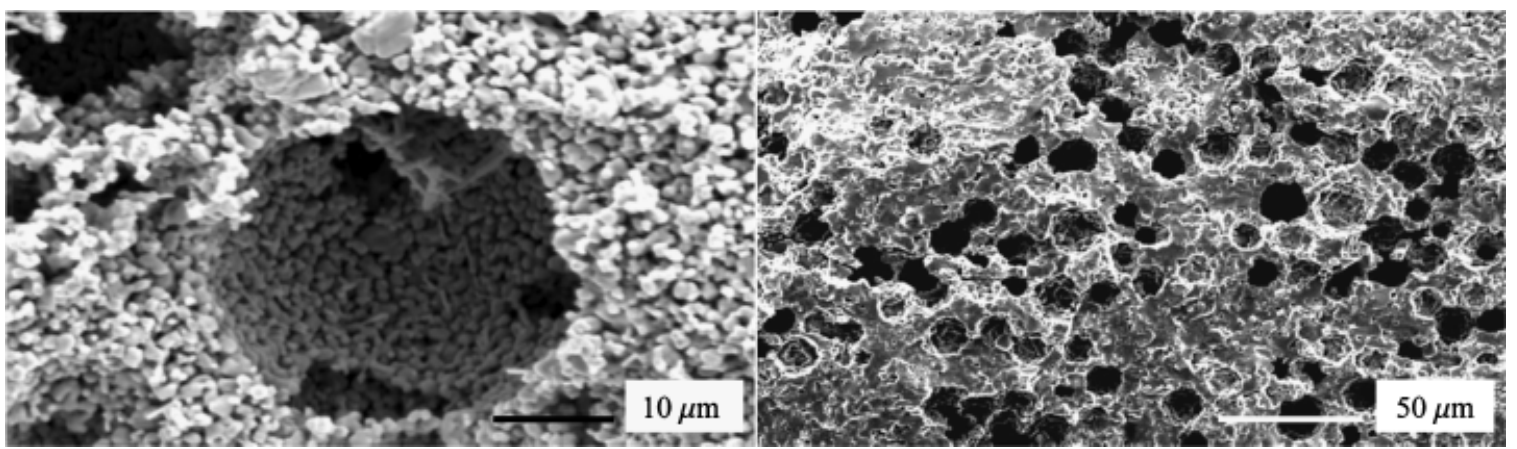

Fig. 3. (Left) Residual carbon pore in a green $\mathrm{TiO}_{2}$ compact after carbon burnout and before sintering. (Right) Fracture surface of sintered calcium magnesium titanates with spherical pores.

the pores. The capacitance was measured at several increments of frequency between 5 and $30 \mathrm{MHz}$. Relative permittivity values were determined from the parallel-plate capacitor geometry. Reported values of permittivity are averages over 5-30 $\mathrm{MHz}$ range, with three repeated measurements per sample, and three identical samples for each porosity.

The relative permittivities of extruded samples were tested using a modified Courtney configuration at the National Institute of Standards (Boulder, CO). These results have been reported in part previously. ${ }^{13}$

\section{Results and Discussion}

\section{(1) Pore Structure}

The pore structure was analyzed using a scanning electron microscope (Philips XL-30, Philips Electronics N.V., Eindhoven, the Netherlands). The induced pores created by the microspheres were validated in the green compact after carbon removal as shown in Fig. 3 (left). Figure 3 (right) shows a fracture surface from a CMT sample. The pore size corresponds to the size of the microspheres $(\sim 15 \mu \mathrm{m})$. Pore distribution appears to be randomly distributed with minimal coalescence, and is surrounded by a well-densified matrix. The use of MFCX in tures. Figure 4 (left) shows a $54 \%$ porous structure fabricated with $20 \mathrm{vol} \% \mathrm{TiO}_{2}$ in the carbon core. Figure 4 (right) shows a very highly porous $(78 \% \mathrm{P})$ solid fabricated with a hollow, cylindrical core. The walls are essentially a single grain in thickness.

\section{(2) Relative Permittivity of $\mathrm{TiO}_{2}$}

Samples of titania with residual micropores were sintered between $1150^{\circ}$ and $1400^{\circ} \mathrm{C}$ using 1-h dwells, which created a porosity that ranged between $5.9 \%$ and $35.4 \%$. Table I presents the relative permittivity and porosity values for the microporous samples sintered at each temperature. The samples with glassy $\mathrm{TiO}_{2}$ produced unique randomly-oriented microcellular struc-

Table I. Residual Sintering Porosity and Permittivity in $\mathrm{TiO}_{2}$

\begin{tabular}{lrr}
\hline Temperature $\left({ }^{\circ} \mathrm{C}\right)$ & \multicolumn{1}{c}{$\% \mathrm{P}$} & \multicolumn{1}{c}{$\varepsilon_{\mathrm{r}}$} \\
\hline 1400 & $5.3 \pm 0.7$ & $100 \pm 2.8$ \\
1350 & $9.1 \pm 1.6$ & $86 \pm 4.0$ \\
1325 & $10.3 \pm 0.4$ & $84 \pm 2.5$ \\
1300 & $11.8 \pm 0.5$ & $82 \pm 3.2$ \\
1250 & $17.5 \pm 1.0$ & $77 \pm 0.1$ \\
1225 & $20.9 \pm 1.5$ & $68 \pm 1.9$ \\
1200 & $24.7 \pm 1.6$ & $62 \pm 0.8$ \\
1150 & $35.9 \pm 0.6$ & $50 \pm 1.2$ \\
\hline
\end{tabular}

carbon porogen achieved higher levels of porosity, with the porosity determined by the volume fraction carbon. All samples were fired at $1200^{\circ} \mathrm{C}$; thus, these pores were embedded inside a matrix that is $24.7 \%$ porous. Table II shows the porosity and relative permittivity for these samples. The total measured porosity values are in good agreement with values expected for volume fraction porogen-induced pores in a matrix with $24.7 \mathrm{vol} \%$ micropores. For example, the samples with $30 \mathrm{vol} \%$ porogen should have $30 \%$ large pores and $(1-0.3)(24.7 \%)$ micropores, or $47.3 \%$ total porosity. The Archimedes density for this case was $46.8 \% \pm 2.0 \%$.

Table II. Glassy Carbon Porogen-Induced Porosities in $\mathrm{TiO}_{2}$

\begin{tabular}{rcc}
\hline$\%$ Porogen & $\% \mathrm{P}$ & $\varepsilon_{\mathrm{r}}$ \\
\hline 5 & $29.8 \pm 0.7$ & $56 \pm 2.1$ \\
10 & $32.4 \pm 1.3$ & $47 \pm 2.8$ \\
20 & $37.8 \pm 2.1$ & $46 \pm 2.9$ \\
30 & $46.8 \pm 2.0$ & $37 \pm 0.6$ \\
40 & $52.7 \pm 2.5$ & $32 \pm 0.5$ \\
50 & $58.3 \pm 1.6$ & $26 \pm 0.9$ \\
\hline
\end{tabular}
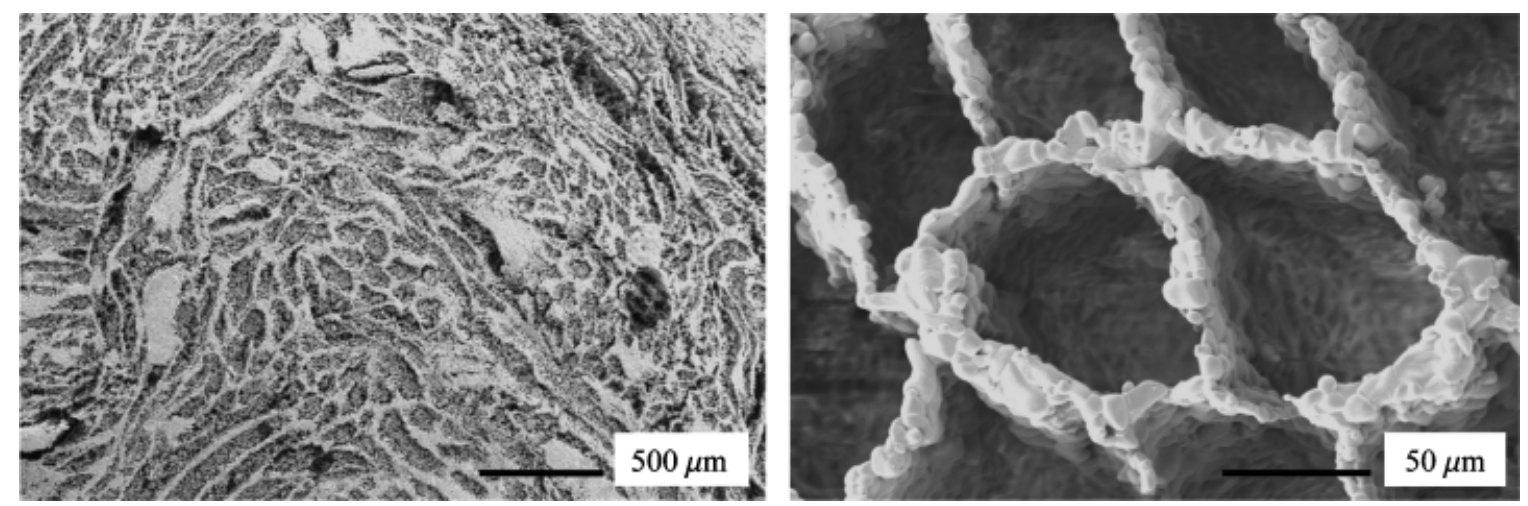

Fig. 4. (Left) Microcellular $\mathrm{TiO}_{2}$ as-fired surface. The microcellular structure shows the dense $\mathrm{TiO}_{2}$ walls surrounding regions of 20 vol\% $\mathrm{TiO}{ }_{2}$. (Right) Dense $\mathrm{TiO}_{2}$ walls surrounding a hollow core. The total porosity is $78 \%$. 


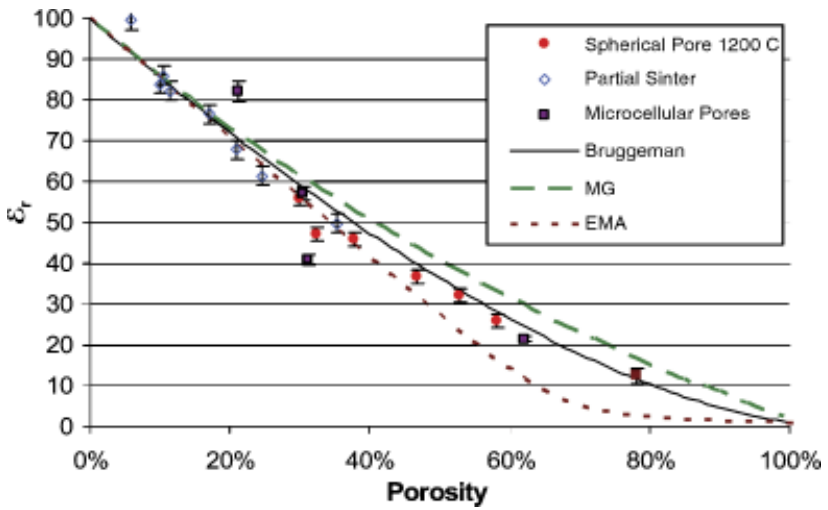

Fig. 5. Dielectric constant data of porous titania compared with Bruggeman, Maxwell-Garnet (MG), and effective medium approximation (EMA) dielectric mixing models. The mixing is between $\mathrm{TiO}_{2}$ $\left(\varepsilon_{\mathrm{r}}=100\right)$ and air $\left(\varepsilon_{\mathrm{r}}=1\right)$.

Figure 5 plots the experimental $\varepsilon_{\mathrm{r}}$ versus porosity against the presented mixing models. Two reference values for porous $\mathrm{TiO}_{2}$ are shown to validate the data set. ${ }^{13}$ It is clear that the experimental data for $\mathrm{TiO}_{2}$ correlate well with the Maxwell-Garnet, EMA, and the Bruggeman power law at levels of porosity less than $20 \%$. Between $20 \%$ and $60 \%$ porosity, the data move away from the EMA and MG and lie closer to the Bruggeman curve. The large errors in MG and EMA are expected at high levels of porosity as their basic assumptions break down. The modeling of the pores as spherical inclusions works well for the residual pores left behind from incomplete densification and the pores formed via the glassy carbon porogen.

The data for the MFCX samples are presented in Table III. Four compositions were created via the extrusion process to vary the levels of porosity. One sample (a) contained a hollow core and three others contained 5, 15, and $20 \mathrm{vol} \% \mathrm{TiO}_{2}$ inside the core (b, c, and d, respectively). Comparison with the Bruggeman model shows good agreement at high levels of porosity, but some significant deviations for two low-porosity samples. These deviations are partially due to departures from the model assumption of spherical pores. Another factor is some degree of nonrandomness. Samples fabricated from chopped fibers pressed together likely undergo a small degree of orientation because of the applied pressure. This can be explained in terms of the processing. There is distortion of the thermoplastic fibers as they are formed into a solid block. This creates inhomogeneous orientation of voids. However, using this technique one can achieve very large decreases in $\varepsilon_{\mathrm{r}}$. The lowest value achieved was at $78 \%$ porosity, yielding an $\sim 90 \%$ reduction in $\varepsilon_{\mathrm{r}}$ from 100 to 12.3

Over the wide porosity range $(5 \%-78 \%)$ for all samples, the relative permittivity depends only on the total porosity, and not on the size or nature of the porosity. As expected from effective medium concepts, samples with similar porosity have similar relative permittivity, whether the pores are large or small.

\section{(3) Relative Permittivity of CMT}

The glassy carbon porogen was also used in the CT-MT- $\mathrm{M}_{2} \mathrm{~T}$ system to achieve porosities in the range of $20-60 \mathrm{vol} \%$. This reduced the permittivity from 27 for the nominally dense sample

Table III. Microcellular $\mathrm{TiO}_{2}$ Porosity and Permittivity

\begin{tabular}{lcc}
\hline Samples & $\% \mathrm{P}$ & $\varepsilon_{\mathrm{r}}$ \\
\hline $\mathrm{a}$ & $78.0 \pm 1.4$ & $12 \pm 1.8$ \\
$\mathrm{~b}$ & $62.0 \pm 4.3$ & $21 \pm 0.6$ \\
$\mathrm{c}$ & $30.3 \pm 0.7$ & $57 \pm 1.7$ \\
$\mathrm{~d}$ & $21.2 \pm 1.2$ & $82 \pm 2.5$ \\
\hline
\end{tabular}

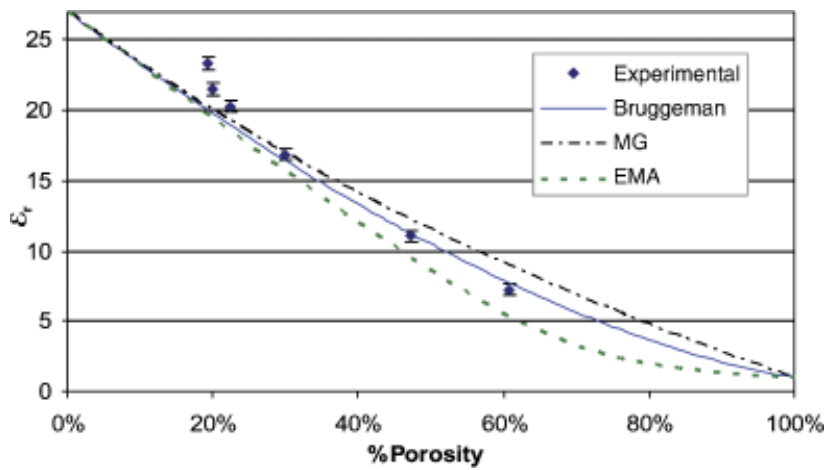

Fig. 6. Dielectric constant of porous calcium magnesium titanate compared with the Bruggeman, effective medium approximation (EMA), and Maxwell-Garnet (MG) effective medium models.

to as low as 7 for the sample with $60 \%$ porosity. Figure 6 shows the permittivity as a function of porosity fit with the MG, EMA, and Bruggeman models. The measured permittivities for the relatively low-porosity $(\sim 20 \%$ P) samples are higher than expected by about $16 \%$. The higher porosity samples are well represented by the Bruggeman model, with the predicted permittivity typically within $6 \%$ of the measured values.

\section{Conclusions}

Partial sintering can achieve porosities as high as $36 \mathrm{vol} \%$, with a reduction of dielectric permittivity up to $50 \%$. The use of carbon microspheres is demonstrated as an effective porogen in $\mathrm{TiO}_{2}$ and a CMT, inducing porosity as high as $60 \mathrm{vol} \%$. Reductions of $\sim 75 \%$ in relative permittivity are possible by using this method. Thermoplastic co-extrusion of titania with carbon powders can produce microcellular $\mathrm{TiO}_{2}$ with porosity up to 78 $\mathrm{vol} \%$. This enables the relative permittivity of $\mathrm{TiO}_{2}$ adjusted from 100 down to 12.3 , a reduction by nearly $90 \%$.

The relative permittivity depends only on the total porosity, and is not sensitive to the size of the pores, following effective medium behavior. The Bruggeman power law model provides a better representation of permittivity over this porosity range than do the Maxwell-Garnet or Landuaer-Bruggeman models. The permittivity of CMT at porosity greater than $30 \%$ can be described accurately with the Bruggeman model.

\section{References}

${ }^{1}$ A. Templeton, X. Wang, S. J. Penn, S. J. Webb, L. F. Cohen, and N. McN Alford, "Microwave Dielectric Loss of Titanium Oxide," J. Am. Ceram. Soc., 83 [1] 95-100 (2000).

${ }^{2}$ V. M. Ferreira, F. Azough, R. Freer, and J. L. Baptista, "The Effect of Cr and $\mathrm{La}$ on $\mathrm{MgTiO}_{3}$ and $\mathrm{MgTiO}_{3}-\mathrm{CaTiO}_{3}$ Microwave Dielectric Ceramics," J. Mater. Res., 12 [12] 3293-9 (1997).

${ }^{3}$ R. Piagi, I.-T. Kim, J. G. Park, and Y. H. Kim, "Preparation of MagnesiumCalcium Titanate Powders by Alkoxide Precursor Method," J. Am. Ceram. Soc., 81 [5] 1361-4 (1998).

${ }^{4}$ A. Mortensen and S. Suresh, "Functionally Graded Metals and MetalCeramics Composites: Part 1, Processing," Int. Mater. Rev., 40, 239-65 (1995).

${ }^{5}$ S. F. Corbin and P. S. Apte, "Engineered Porosity Via Tape Casting, Lamination and the Percolation of Pyrolyzable Particulates," J. Am. Ceram. Soc., 82 [7] 1693-701 (1999).

${ }^{6}$ E. A. Vasilyeva, L. V. Morozova, A. E. Lapshin, and V. G. Konakov, "Ceramic Materials with Controlled Porosity," Mater. Phys. Mech., 5, 43-8 (2002).

${ }^{7}$ K. Maexa, M. R. Baklanov, D. Shamiryan, F. Iacopi, S. H. Brongersma, and Z. S. Yanovitskaya, "Low Dielectric Constant Materials for Microelectronics," J. Appl. Phys., 93, 11 (2003).

${ }^{8}$ L. Favenneca, V. Jousseaumeb, V. Rouessacc, F. Fusalbaa, J. Durandc, and G Passemarda, "Porous Extreme Low k (ELk) Dielectrics Using a PECVD Porogen Approach," Mater. Sci. Semicond. Process., 7, 277-82 (2004).

${ }^{9}$ S. Seraji, Y. Wu, M. Forbess, S. J. Limmer, T. Chou, and G. Cao, "Sol-GelDerived Mesoporous Silica Films with Low Dielectric Constants," Adv. Mater., 12, 22 (2000)

${ }^{10} \mathrm{G}$. Kiziltas, D. Psychoudakis, J. L. Volakis, and N. Kikuchi, "Topology Design Optimization of Dielectric Substrates for Bandwidth Improvement of a Patch Antenna," IEEE Trans. Antennas Propag., 51 [10, Part 1] 2732-43 (2003). 
${ }^{11}$ Y. H. Koh, J. W. Halloran, G. Kiziltas, D. Psychoudakis, and J. L. Volakis, "Thermoplastic Green Machining of Textured Dielectric Substrate for Broadband Miniature Antenna," J. Am. Ceram. Soc., 88 [2] 297-302 (2005).

${ }^{12}$ W. J. Chappell and X. Gong, "Wide Bandgap Composite EBG Substrates," IEEE Trans. Antennas Propag., 51 [10, Part 1] 2744-50 (2003).

${ }^{13}$ X. Gong, W. H. She, E. J. Hoppenjans, Z. N. Wing, R. G. Geyer, J. W. Halloran, and W. J. Chappell, "Tailored and Anisotropic Dielectric Constants Through Porosity in Ceramic Composites," IEEE Trans. Microwave Theory Tech., 53 [11] 3638-47 (2005).

${ }^{14}$ D. Kingery, H. K. Bowen, and D. Uhlmann, Introduction to Ceramics, 2nd Edition, John Wiley \& Sons Inc, New York, 1976.
${ }^{15} \mathrm{O}$. Wiener, "Die Theorie des Mischkorpers fur das Feld der staionaren Stromung," Der Abhandlungen der Mathematisch-Physischen Klasse der Konigl. Sachsischen Gesellschaft der Wissenschaften Leipzig, 32, 509-604 (1912).

${ }^{16}$ P. S. Neelakanta, Handbook of Electromagnetic Materials: Monolithic and Composite Versions and Their Applications. Research Studies Press, Boca Raton, FL, 2003.

${ }^{17}$ D. Stroud, "The Effective Medium Approximations: Some Recent Developments," Superlattices Microstruct., 23 [3/4] 567-73 (1998).

${ }^{18}$ D. Stroud, "Generalized Effective-Medium Approach to the Conductivity of an Inhomogeneous Material," Phys. Rev. B, 12, 3368 (1975).

${ }^{19} \mathrm{C}$. V. Hoy, A. Barda, M. L. Griffith, and J. W. Halloran, "Microfabrication of Ceramics by Co-Extrusion," J. Am. Ceram. Soc., 81 [1] 152-8 (1998). 